\title{
Polarized solid target material developments at Bonn and Bochum
}

\author{
S. Goertz* \\ Physics Institute, University of Bonn, Nussallee 12, 53115 Bonn, Germany \\ E-mail: goertz@physik.uni-bonn.de
}

\author{
A. Berlin ${ }^{b}$, M. Bornstein ${ }^{a}$, H. Dutz ${ }^{a}$, J. Herick ${ }^{b}$, S. Reeve ${ }^{a}$, G. Reicherz ${ }^{b}$, S. Runkel ${ }^{a}$ \\ ${ }^{a}$ Physics Institute, University of Bonn, Nussallee 12, 53115 Bonn, Germany \\ ${ }^{b}$ Institute for Experimental Physics I, Ruhr University Bochum, 44780 Bochum, Germany
}

\begin{abstract}
There is a long tradition of polarized solid targets at the Bonn university already starting in the late 1960s with first target asymmetry experiments at the former Bonn $2.5 \mathrm{GeV}$ synchrotron done by Karl-Heinz Althoff and coworkers. An early milestone set in the field of polarizable solid target materials was the development of irradiated d-ammonia in the early 1980s by W. Meyer. In the subsequent years the preparation process as well as the polarization behavior of this material were further optimized. In 1995 the target material expertise moved to Bochum, when W. Meyer joined the Ruhr University as a professor. Under his leadership the target material lithium deuteride was further developed with the result of the preparation of about one liter highly polarizable material for the COMPASS experiment at CERN. Its first data taking period was dedicated to the further measurement of the spin dependent structure function of the deuteron $\mathrm{g}_{1}^{d}$ followed by the $\Delta \mathrm{G}$ program measuring the gluon spin contribution to the nucleon. The investigations into this target material gave new insights into the role of paramagnetic centers in the dynamic nuclear polarization process. As a result several new developments were initiated like the trityl doped alcohols and diols as well as irradiated hydrocarbons. Investigations into the very promising materials polyethylene and polypropylene are still ongoing at the accelerator facilities of the Bonn university.
\end{abstract}

XVIth International Workshop in Polarized Sources, Targets, and Polarimetry, PSTP2015,

14-18 September 2015,

Bochum, Germany

${ }^{*}$ Speaker. 


\section{Introduction}

Within the recent decades polarized solid targets as well as polarized particle beams evolved into indispensable tools in order to explore the structure of the proton and the neutron. Single and double polarization measurements were performed with a variety of different particles beams, among them not only experiments with polarized electrons and real photons. Protons, antiprotons and even polarized muons have been used so far. The Physics Institute of the Bonn university was one of the pioneering institutions in this research field worldwide. Very soon after the commissioning of the $2.5 \mathrm{GeV}$ synchrotron in 1967 it became more and more apparent that only experiments with defined spin states of the colliding particles will allow a deeper understanding of the nature of the nucleons. Thus researchers put a lot of effort in the development of the respective technologies initially being the measurement of the target recoil polarization and later on the technique of polarized solid targets. One of the most important components of a polarized solid target is the target material itself, which has to fulfill several requirements combined in the same substance (see section 2). The choice and the accurate preparation of a polarizable solid state target material is still an actual research area done at a handful of laboratories in the world. Together with the universities of Bochum and Mainz the Bonn university is still one of the centers of competence in the field of polarized solid targets in Germany and is home of the Electron Strechter Anlage (ELSA), at which experiments with polarized photon beams and polarized proton/neutron targets are still performed and contribute to the world data base. For a detailed and comprehensive overview over this research field the reader is referred to a dedicated review article [1].

\section{Parameters characterizing suitable target materials}

In order to correctly judge the influence of the material properties on the accuracy of an experiment with a polarized target, the target asymmetry $A$ and its respective error $\Delta A$ shall be considered. The target asymmetry observable $A$ may be calculated from the counting rate asymmetry

$$
\varepsilon=\frac{N \uparrow-N \downarrow}{N \uparrow+N \downarrow}=: \frac{\Delta N}{N_{t o t}}
$$

via the relation

$$
A=\frac{1}{P_{T}} \cdot \frac{1}{f} \cdot \varepsilon
$$

In this formula $P_{T}$ denotes the target polarization and $f$ the so-called dilution factor, being the fraction of polarizable nucleons to the sum of all nucleons in the material. The error expansion of $A$ results in

$$
\left(\frac{\Delta A}{A}\right)^{2}=\left(\frac{\Delta P_{T}}{P_{T}}\right)^{2}+\left(\frac{\Delta f}{f}\right)^{2}+\left(\frac{\Delta \varepsilon}{\varepsilon}\right)^{2} \simeq\left(\frac{\Delta \varepsilon}{\varepsilon}\right)^{2},
$$

where the latter identity is approximately valid, since the target polarization as well as the dilution factor can be determined quite accurately. Since the counting rate asymmetry is usually a small number, its error can be written as $\Delta \varepsilon \simeq 1 / \sqrt{N_{\text {tot }}}$. Using these relations the measuring time $t_{\text {meas }}$ needed to obtain a certain acceptable error in the asymmetry observable $A$ may be expressed as follows:

$$
t_{\text {meas }} \propto N_{\text {tot }} \simeq \frac{1}{P_{T}^{2}} \cdot \frac{1}{f^{2}} \cdot \frac{1}{\Delta A^{2}}
$$


Taking into account the luminosity $L=I \cdot n_{T}$ being the product of the beam intensity $I$ and the target thickness $n_{T}$ as well as the spin independent cross section $\sigma_{t o t}$ of the specific reaction, one may additionally use the relation

$$
N_{\text {tot }}=L \cdot \sigma_{\text {tot }} \cdot t_{\text {meas }}
$$

in order to find a more detailed expression for the needed measuring time:

$$
t_{\text {meas }}=\frac{1}{\sigma_{t o t}} \frac{1}{\Delta A^{2}} \cdot \frac{1}{L f^{2} P_{T}^{2}}=: \frac{1}{\sigma_{t o t} \Delta A^{2}} \cdot \frac{1}{L \cdot \mathrm{FoM}}
$$

From this formula it is immediately clear that as far as the polarized target is concerned the dilution factor $f$ and the average target polarization $P_{T}$ have the largest impact on the target Figure of $\underline{\text { Merit }}$ FoM. In contrast to these numbers, which appear quadratically in the Figure of Merit, the FoM only depends linearly on the target thickness, i.e. on the product of material density and target length.

Besides a FoM number as high as possible, in the case of an experiment performed with a high intensity beam there is at least one further criterion, which decides the suitability of the target material in question. This is the so-called radiation hardness, i.e. the resistance of the material against a reduction of the available polarization due to the experimental particle beam. When on the other hand the experiment is of the frozen spin type and thus operating with a low intensity beam, reasonable build-up and relaxation times of the polarization are important, in order to ensure a decent relation between repolarization and data taking periods. Eventually there are also practical aspects like the simplicity in handling and the doping of the material (see section 3) as well as a good reproducibility of the preparation procedure in general.

\section{The basic concept of Dynamic Nuclear Polarization}

The difficulty in obtaining a substantial nuclear spin polarization originates from the extremely small sizes of the nuclear magnetic moments. Even at temperature well below $1 \mathrm{~K}$ and at magnetic fields of several Tesla, the polarization of an ensemble of protons will not exceed the level of some percent at best. Table 1 summarizes the values for protons, deuterons and electrons under the usual

\begin{tabular}{c|c|c}
$P_{p}[\%]$ & $P_{d}[\%]$ & $P_{e}[\%]$ \\
\hline 0.25 & 0.05 & 93
\end{tabular}

Table 1: Thermal equilibrium polarization values of protons, deuterons and electrons at a temperature of $1 \mathrm{~K}$ and a magnetic field of $2.5 \mathrm{~T}$

conditions of $1 \mathrm{~K}$ and $2.5 \mathrm{~T}$. The basic mechanism of the DNP (Dynamic Nuclear Polarization) process is the transfer of the high electron polarization to the nuclear spin system via exposing the spin system to a microwave field with frequencies slightly off the electron Larmor frequency. Details concerning the DNP mechanisms would go far beyond the scope of this article. A comprehensive summary may be found in [2]. The above mentioned process obviously assumes the presence of unpaired electron spins, i.e. of a certain amount of paramagnetic impurities in the material. Since almost all chemically stable substances are diamagnetic by nature, one has to add these impurities 
artificially. This procedure - called doping - can be done in different ways: Whereas in earlier times the common method was to produce the paramagnetic impurities (radicals) in the (liquid) material itself by certain chemical reactions [3], there are two methods, which are prevalent nowadays:

1. Admixture of a certain amount of chemically stable radicals to the substance

2. Creation of paramagnetic defects by irradiating the substances with ionizing radiation

Method no.1 is usually chosen, when the target material is liquid at room temperature and thus may serve as a solvent for the radical. If on the other hand the material is solid at room temperature, option no. 2 is the standard method, although it has been applied successfully to frozen liquids in the past as well [4].

\section{Early achievements in polarized solid target materials}

Based on the "Overhauser Effect" found in 1953 [5] the "Solid State Effect" was discovered in 1957 by C. D. Jeffries as a mechanism that provides the possibility of a polarization transfer between different magnetic moments using coupled spin transitions [6]. It was demonstrated only one year later by A. Abragam in the material ${ }^{6} \mathrm{LiF}$ for the first time [7]. In this material it is the fluorine nucleus with its relatively large magnetic moment, which assumes the role of the unpaired electron and thus serves as the source of polarization for the adjacent lithium nuclei. Just within the next few years many substances were found able to produce nuclear polarizations in the order of a few percent.

The breakthrough in the search for a substance, which was able to deliver polarization values suitable for first particle physics experiments came in 1962 with the discovery of the material Nd:LMN $\left(\cdot 24 \mathrm{H}_{2} \mathrm{O}\right)$, the Lanthanium Magnesium Nitrate doped with paramagnetic Nd-Ions [8]. Until 1968 this material was used in more than 40 experiments with polarization up to $70 \%$ at temperatures of about $1 \mathrm{~K}$ and magnetic fields around $2 \mathrm{~T}$. LMN was a perfect example of a material polarizing according to the "resolved solid state effect", which predicts a high degree of polarization even under those very moderate temperature and field conditions.

The next huge step forward was made by M. Borghini, S. Mango, O. Runolfson, J. Vermeulen and D. A. Hill in 1966 with the introduction of the alcohols - particularly of butanol $\left(\mathrm{C}_{4} \mathrm{H}_{9} \mathrm{OH}\right)$ as target materials [9],[10],[11]. There are two big advantages of this material over LMN: Firstly, butanol possesses a much larger radiation hardness being more than two orders of magnetude higher than that of LMN. This enabled the use of more intense particle beams. Secondly, the content of polarizable protons (dilution factor) is $13.5 \%$ in butanol compared to only $3.1 \%$ in LMN leading to an enormous improvement in the efficiency of the polarized target. Both improvements together with maximum polarizations of $40 \%$ and $70-80 \%$ using a ${ }^{4} \mathrm{He}-$ and a ${ }^{3} \mathrm{He}-$-evaporation cryostat, respectively, allowed a new class of experiments with a greatly enhanced precision.

Experiments of this kind using a high intensity photon beam from the $2.5 \mathrm{GeV}$ synchrotron were already performed in Bonn in the years 1971-1973 with the measurement of the $\pi^{+}$photoproduction off polarized protons under fixed CM angles [12]. In the year 1971 the first polarized neutron target became available with the development of the fully deuterated version of butanol $\left(\mathrm{C}_{4} \mathrm{D}_{9} \mathrm{OD}\right)$. Deuteron polarizations of $22 \%$ were obtained in a $2.5 \mathrm{~T}$ magnetic field using a ${ }^{3} \mathrm{He}-$ evaporation cryostat producing temperatures around $0.5 \mathrm{~K}[13]$ and of $26 \%$ in a $5.0 \mathrm{~T}$ magnetic 
field using an ${ }^{4} \mathrm{He}$-evaporation cryostat operating at $1 \mathrm{~K}$ [14]. With this new material the experiments at the Bonn $2.5 \mathrm{GeV}$ synchrotron could be extended to the photoproduction of negative pions off polarized neutrons in the subsequent years [15].

\section{The 1980s: Irradiated frozen ammonia}

Although in the meantime a nearly completely polarized proton target was available with $\mathrm{Cr}(\mathrm{V})$ doped 1,2-propanediol using a ${ }^{3} \mathrm{He} /{ }^{4} \mathrm{He}$ dilution refrigerator [16], the next big leap in target material science was done with the development of ammonia as a polarizable target material. In 1979 T. O. Niinikoski and J.-M. Rieubland managed to polarize irradiated frozen $\mathrm{NH}_{3}$ to more than $90 \%$ using again a dilution refrigerator [17].

Very soon after the introduction of $\mathrm{NH}_{3}$ also its deuterated counterpart $\mathrm{ND}_{3}$ was investigated with respect to applications as polarized deuteron or neutron target materials. These studies, as well as further investigations into the polarization behavior and the radiation resistance of $\mathrm{NH}_{3}$, were performed by W. Meyer et al. of the university of Bonn [18]. The samples were frozen either quickly by dropping the liquid ammonia into a liquid nitrogen bath or slowly in form of a crystalline block, which was subsequently crushed into chips of the desired size (1-2mm side length). The material solidified in such ways was then irradiated in a special irradiation cryostat [19] under liquid argon temperatures using the $20 \mathrm{MeV}$ electron beam of the Bonn injection linac. EPR spectra taken after the irradiation showed clear evidence of being dominated by the $\dot{\mathrm{NH}_{2}}$ and the $\mathrm{ND}_{2}$ radical, respectively. These investigations exhibited remarkable results in several aspects:

1. Preirradiated $\mathrm{NH}_{3}$ could be polarized up to $37 \%$ in a magnetic field of $2.5 \mathrm{~T}$ and at a temperature of about $1 \mathrm{~K}$, being the standard conditions available at that time.

2. After a further "in situ" irradiation with an high energy electron beam at $1 \mathrm{~K}$ the polarization dropped to an asymptotic value of about $25 \%$ accompanied by a greatly widened frequency curve (polarization vs. applied microwave frequency).

3. The same asymptotic polarization value $(\sim 25 \%)$ is reached, when virginal $\mathrm{NH}_{3}$ (without any preirradiation) is exposed to the high energy electron beam at $1 \mathrm{~K}$ at the same accumulated dose as in (2).

4. Virginal material, which was periodically exposed to the high energy electron beam and subsequently annealed, even reached polarization values as high as $47 \%$ directly after the respective annealing process.

5. In $\mathrm{ND}_{3}$ the influence of the in situ irradiation has an even stronger effect on its polarization behavior. After about $4 \times 10^{14}$ high energy electrons per $\mathrm{cm}^{2}$ the maximum polarization obtained was more than $7 \%$ compared to an initial value of only $3.5 \%$ from exclusively preirradiated material.

Concerning its properties as a polarized target material ammonia possesses two decisive advantages compared to the alcohols and diols used so far: (1) A substantially larger dilution factor of $17.3 \%$ as well as (2) a further extended radiation hardness by another order of magnitude both compared 
to the standard proton target material at that time, the h-butanol, with a dilution factor of $13.5 \%$ and a radiation hardness of some $10^{14}$ part. $/ \mathrm{cm}^{2}$. Additionally - as it was already seen in butanol - the reduction in the available polarization due to damage by the experimental beam could be reversed by an annealing process. These properties make frozen ammonia in all its isotopic compositions $\left({ }^{14} \mathrm{NH}_{3},{ }^{15} \mathrm{NH}_{3},{ }^{14} \mathrm{ND}_{3}\right.$ and the very expensive $\left.{ }^{15} \mathrm{ND}_{3}\right)$ a nearly perfect target material for precision particle physics experiments. This is true under both experimental conditions, (1) in experiments with intense particle beams, where due to the needed cooling power the target temperature is restricted to around $1 \mathrm{~K}$ as well as (2) in frozen spin experiments using dilution refrigerators.

Since its discovery polarized $\mathrm{h}$ - and d-ammonia has been used in many experiments worldwide spanning from investigations into the properties of the deuteron [20] up to the measurement campaign searching for the spin composition of the nucleon with experiments at SLAC (E143) and at CERN (EMC, SMC and COMPASS) during the last three decades.

\section{The 1990s: Irradiated lithium hydrides}

The $\mu$ program of the COMPASS experiment at CERN started in the year 2002 with the measurement of inclusive and semi-inclusive double-spin asymmetries, in order to disentangle the quark and the gluon spin content of the nucleon. In order to maximize the efficiency of the experiment it was proposed to use ${ }^{6} \mathrm{LiD}$ as the polarized target material. In a very naive picture, in which the ${ }^{6} \mathrm{Li}$ nucleus is imagined as an object composed of an $\alpha$ particle and a deuteron, one expects a dilution factor as high as $50 \%$. More refined models of the ${ }^{6} \mathrm{Li}$ indicate after all around $45 \%$ [21]. However since it is the effective polarization, which determines the target efficiency, the achievable polarization value is as equally important as the dilution factor in this regard.

There is a quite long history of efforts to dynamically polarize the lithium hydrides going back to the years 1978-1980, when these materials were used to study magnetic ordering processes and were subsequently proposed as polarized target materials [22],[23]. In the 1980's and beginning 1990's a quite intense period of research in these materials followed again carried out by the Saclay target group [24],[25],[26]. Within these investigations it was found that the temperature, under which the radiation doping takes place, is a crucial parameter in order to maximize the achievable nucleon polarization.

In 1990 it was W. Meyer of the university of Bonn, who had the idea to further continue these studies at the Bonn electron accelerator facility ELSA. In doing so a diploma thesis was awarded in order to construct a first version of a special irradiation cryostat needed to deliver the variable and reliable temperature conditions during the irradiation at the injection linac 1 of ELSA. In the framework of a subsequent $\mathrm{PhD}$ thesis first irradiations of ${ }^{6,7} \mathrm{LiH} /{ }^{6} \mathrm{LiD}$ samples were performed at Bonn using an improved version of the irradiation cryostat. It was found that there is a well defined irradiation temperature $(\sim 185 \mathrm{~K})$, which leads to optimum polarization results in all isotopic compositions in agreement with the previous studies by the Saclay group. Furthermore extended EPR studies showed that the paramagnetic center that is formed during irradiation is the "F-center", a defect, which is well known from the radiolysis of alkali halides [27].

When W. Meyer accepted a call as a professor to the Ruhr University Bochum in 1995, the expertise in the field of target material research moved with him to the new institution. In the following years the Bochum target group were able to further optimize the polarization properties 
of the lithium hydrides in particular those of ${ }^{6} \mathrm{LiD}$. Within an another $\mathrm{PhD}$ thesis as much as 1 liter of this material was synthesized from the elements and irradiated at the Bonn linac 1. Besides a further confirmation of the fact that the irradiation temperature plays an important role, it was observed that the ${ }^{6} \mathrm{Li}$ isotopic purity has a major impact on the maximum available polarization as well. To summarize one may say that a further decade (1990-2000) of additional research was necessary to transform this material from an intriguing idea to a reliable target material, which keeps its performance even when produced in large amounts. From the year 2000 on about one liter crushed chips of this material produced in the way described above was delivered to CERN and polarized routinely to about $55 \%$ in the target cryostat of the COMPASS experiment [28].

Whereas the COMPASS polarized target operates in the so-called continuous mode, i.e. the DNP process is also maintained during data taking (longitudinal spin orientation only), a ${ }^{6} \mathrm{LiD}$ sample of the same production batch was also used in a frozen spin experiment, namely in the 2002 run of the GDH experiment at ELSA measuring the Gerasimov-Drell-Hearn sum rule on the neutron [29].

${ }^{6} \mathrm{LiD}$ outperforms all other target materials in at least two ways: (1) It is not only the unmatched effective polarization already mentioned, but there is another big advantage: (2) It offers a factor of five in terms of radiation hardness compared to the "second best choice", ammonia. The lithium hydrides are able to accept about $10^{16}$ part./ $\mathrm{cm}^{2}$ of experimental beam before its original state has to be restored via an annealing process [30]. Thus, the material is predestined for high intensity experiments, such as the E155 experiment at SLAC, in which it was already used in the year 1998.

\section{The 2000s: Trityl doped target materials}

In the beginning 2000s the situation was as follows:

Proton target materials: The usual target materials in their normal isotopic composition (hydrogenated) could be polarized almost completely under all relevant experimental conditions (frozen spin technique and/or continuous polarization under intense particle beams).

Deuteron Target materials: Deuteron polarizations available from solid state target materials were limited to around $50 \%$.

The essential difference between the deuteron and the proton with respect to their achievable spin polarizations is the respective magnetic moment $\mu_{p, d}$ being more than three times lower for the deuteron compared to the proton. Under the assumption of a unique temperature $\mathrm{T}_{s s}$ of the electron non Zeeman reservoir the DNP process via a thermal contact of the nuclear Zeeman to the electron non Zeeman reservoir leads to the following approximate expression of the proton/deuteron polarization:

$$
P_{p, d} \simeq\left(\frac{2}{3}\right)_{d} \frac{\mu_{p, d} B}{k T_{s s}}
$$

The factor (2/3) has to be considered only in the deuteron case. Although eq. 7.1 applies strictly speaking only to the so called high temperature limit, the general dependencies are true for the low temperatures of a target cryostat as well. The only chance to improve the situation for the deuterons 
is to find a method to further reduce the spin-spin temperature $\mathrm{T}_{s s}$. The minimum achievable $\mathrm{T}_{s s}$ is directly related to the width of the electron paramagnetic (EPR) resonance line. Thus the task was and still is to find new doping methods for preferably all existing deuterated target materials, which produce paramagnetic centers with narrow EPR lines, in order to reduce $\mathrm{T}_{s s}$ as far as possible. A short survey of the theoretical background of the underlying EST theory can be found in [31]. Triggered by the results of a swedish research group [32] (see also below), the Bochum target group found with the chemically stable "trityl" radical a substance, which turned out to be particularly suitable as a dopant for the deuterated versions of butanol and propanediol. In the year 2003 both d-butanol and d-propanediol were polarized up to $80 \%$ in a ${ }^{3} \mathrm{He} /{ }^{4} \mathrm{He}$ dilution refrigerator at a magnetic field of $2.5 \mathrm{~T}$ [4].

During the subsequent years the trityl doped d-butanol was used as a polarized target material for hadron physics experiments of (1) the A2 collaboration at MAMI (Mainz) in the years 2003/2015 [33] and of (2) the Crystal Barrel collaboration at ELSA in the year 2010. Both experiments are using the frozen spin technique meaning that every spin manipulation and particularly the time, in which the target sample can be repolarized, is limited due to efficiency considerations. Nevertheless deuteron polarizations of more than $70 \%$ have been obtained at the Mainz A2 experiment under these circumstances.

Butanol and propanediol are not the only substances, which greatly benefit from the new radical. Also polymer materials have been investigated in this regard. The current situation for dynamically polarized deuterated polymer target materials with trityl radicals is that in deuterated polystyrene, chemically doped with the trityl radical Finland D36, polarization values up to $60 \%$ have been achieved in a $5 \mathrm{~T}$ magnetic field using a dilution refrigerator [34].

In the last two decades a completely different application of DNP was discovered, being the "hyperpolarization" of nuclei in medical imaging. During a MRT scan the NMR signal from a ${ }^{13} \mathrm{C}$ enriched substance is observed, which was previously dynamically polarized, liquefied and injected into a living creature [32]. The Bochum polarized target group also participated in these investigations with a measurement of dynamically polarized trityl doped pyruvic acid in magnetic fields up to $5.0 \mathrm{~T}$, in which values around $80 \%$ could be observed [35]. This very high ${ }^{13} \mathrm{C}$ polarization is certainly a result of the small EPR-linewidth of the trityl radical even at high field [36].

\section{The 2010s: Irradiated polyethylene and polypropylene}

Since about two decades a new class of solid state target materials came more and more into the focus of the particle and target physicists being the polymer materials. Under their huge variety it is essentially the $\mathrm{CH}_{2}$, which is of particular interest due to its highest possible dilution factor compared to all other polymers. It exists in two manifestations polyethylene and polypropylene. The particular charm of these materials arises from the fact that both are solid at room temperature and easily shapeable. Thus one may think of almost arbitrary target geometries starting from simple thin foils up to any manufacturable shapes.

The special challenge in the preparation of $\mathrm{CH}_{2}$ as a polarized target material is to find an accurate doping method. Beside a thermal diffusion method, in which thin foils of this material may be doped with the TEMPO free radical at elevated temperatures [37],[38], it is the irradiation with ionizing radiation, which appears to be the most promising technique. In the beginning 2000's the 
Bochum polarized target group made some efforts in irradiating polyethylene pellets with electrons from the $20 \mathrm{MeV}$ electron linac (linac 1) of the Bonn accelerator facility ELSA. During irradiation the pellets were immersed in a liquid argon bath at a temperature of $87 \mathrm{~K}$. The samples produced in this way were investigated with regard to the X-band EPR signals from the produced paramagnetic defects as well as polarized in the Bochum vertical $1 \mathrm{~K}$ evaporation cryostat at a magnetic field of $2.5 \mathrm{~T}$. Some percent of proton polarization could be achieved from samples having the usual electron spin concentration of several $10^{19} / \mathrm{cm}^{3}$. The DNP active paramagnetic center itself was determined to be the prominent alkyl-radical representing a hydrogen vacancy somewhere along the $\mathrm{CH}_{2}$ chain. Already at that time it had been observed that a subsequent exposition of the samples to room temperature for some minutes drastically changes the polarization behavior towards much higher maximum polarizations but also towards much longer polarization build up times. With this in mind a new research project was started in the year 2012 in the framework of a $\mathrm{PhD}$ thesis at the Bonn polarized target group. The main aim of this work is to clarify the role of the temperature, to which polyethylene and polypropylene samples are exposed during or after the electron irradiation with regard to their polarization behavior. Details of the ongoing research in this field can be found in the corresponding article of these proceedings [39]. The situation for radiation doped deuterated polyethylene is discussed in these proceedings as well [40].

\section{Summary}

Currently there is a tradition of more than 30 years of target material research at the universities of Bonn and Bochum, which is closely linked to W. Meyer as the prime mover. The development of $\mathrm{ND}_{3}$ as well as his ideas, on how to improve the preparation procedure of the lithium hydrides, came about in his time as the leader of the Bonn polarized target group until 1995. In the same year W. Meyer was appointed professor at the Ruhr University Bochum and the target material research activities also went with him to the new institution. In the year 2000 the investigations into the lithium hydrides were crowned with the preparation of a large amount of highly polarizing ${ }^{6} \mathrm{LiD}$ for the COMPASS experiment at CERN. The following years concentrated on the further development of deuterated target materials, mainly of d-butanol. Triggered by results from the ${ }^{6} \mathrm{LiD}$ studies it appeared to be clear that the problem of the 'low' deuteron polarization may be solved by choosing a doping method better suited for deuterated materials. This was finally found by the use of trityl radicals as chemical dopants for selected organic target materials. Current activities, which are still performed in close collaboration of the Bonn and the Bochum polarized target groups, address the polymer materials polyethylene and polypropylene as very interesting alternatives to the alcohol and diol materials used so far for polarized experiments in hadron physics. The outstanding features of these materials are their temperature stability as well as the possibility to produce targets in nearly any physical shape from them. Investigation into the optimization of the radiation doping at the $20 \mathrm{MeV}$ Bonn electron linac are still ongoing and look very promising.

\section{References}

[1] S. Goertz, W. Meyer and G. Reicherz, Progress in Particle and Nuclear Physics 49 (2002) 403

[2] A. Abragam, M. Goldmann, Rep. Prog. Phys 41 (1978) 395 
[3] M. Borghini, Choice of materials for polarized proton targets, Yellow Report CERN 66-3 (1966)

[4] S. Goertz et al., Nucl. Instr. and Meth. A 526 (2004) 43

[5] A. W. Overhauser, Phys. Rev. 92 (1953) 411

[6] C. D. Jeffries, Phys. Rev. 106 (1957) 164; Phys. Rev. 117 (1960) 1056

[7] A. Abragam, W. G. Proctor, Compt. Rend. Acad. Sci. (Paris) 246 (1958) 2253

[8] T. J. Schmugge and C. D. Jeffries, Phys. Rev. Lett. 9 (1962) 268

[9] M. Borghini, S. Mango, O. Runolfson and J. Vermeulen, Proc. Int. Conf. on Polarized Targets an Ion Sources (Saclay 1966) 387

[10] S. Mango, O. Runolfson and M. Borghini, Nucl. Instr. and Meth. 72 (1969) 45

[11] D. A. Hill et al., Phys. Rev. Lett. 23 (1969) 460

[12] K.H. Althoff et al., Nucl. Phys. B 53 (1973) 9

[13] M. Borghini and K. Scheffler, Nucl. Instr. and Meth. 95 (1971) 93

[14] M. Borghini, A., Masaike, K. Scheffler and F. Udo, Nucl. Instr. and Meth. 97 (1971) 577

[15] K.H.Althoff et al., Nucl. Phys. B 96 (1975) 497

[16] W. de Boer, T. O. Niinikoski, Nucl. Instr. and Meth. 114 (1974) 495

[17] T. O. Niinikoski and J.-M. Rieubland, Phys. Lett. 72A (1979) 141

[18] U. Haertel, O. Kaul, W. Meyer, K. Rennings, E. Schilling, Proc. High-Energy Phys. with Pol.Beams and Pol.Targets, Lausanne 1980, Eds.C. Joseph, J. Soffer, Birkhäuser Verlag Vol.38, 447

[19] S. Brown et al., Proc. $4^{\text {th }}$ Int. Workshop on Polarized Target Materials and Techniques, Bonn 1984, Ed. W. Meyer, 66

[20] K.H. Althoff et al., Z. Phys. C 26 (1984), 175

[21] N. W. Schellingerhout et al., Phys. Rev. C 48 No. 6 (1993) 2714

[22] Y. Roinel, V. Bouffard and P. Roubeau, J. Physique 39 (1978) 1097

[23] A. Abragam, V. Bouffard, Y. Roinel and P. Roubeau, J. Physique Lettres 41 (1980) 309

[24] P. Chaumette et al., Proc. $4^{\text {th }}$ Int. Workshop on Polarized Target Materials and Techniques, Bad Honnef (Germany) 1984, Ed. W. Meyer, 81

[25] G. Durand et al., Proc. $8^{\text {th }}$ Int. Symposium on High Energy Spin Physics, Minneapoli 1988, Am. Inst. of Physics, Conf. Proc. 187 Vol. 21275

[26] G. Durand and J. Ball, Progress Report on Polarizable Lithium Hydrides, CEN Saclay (France) Internal Report (1993)

[27] S. Goertz et al., Nucl. Instr. and Meth. A 356(1995) 20

[28] K Kondo et al., Nucl. Instr. and Meth. A 526 (2004) 70

[29] Ch. Rohlof and H. Dutz, Nucl. Instr. and Meth. A 526 (2004) 126

[30] S. Bültmann et al., Nucl. Instr. and Meth. A 425 (1999) 23

[31] S. Goertz et al., Nucl. Instr. and Meth. A 526 (2004) 28

[32] J. H. Ardenkjear-Larsen et al., Proc. Natl. Acad. Sci. USA (2003) 10158 
[33] A. Thomas, The new frozen spin target at MAMI, Conference Proceedings The 20th International Symposium on Spin Physics (SPIN2012) JINR, Dubna September 17âĂŞ22, 2012, Physics of Particles and Nuclei, Vol. 44, No. 6 (2013) 964

[34] L. Wang et al. Nucl. Instr. and Meth. A 729 (2013) 36

[35] W.Meyer et al. Nucl. Instr. and Meth. A 631 (2011) 1

[36] J. Heckmann et. al. Phys.Rev. B 74134418 (2006)

[37] B. van den Brandt et al., Nucl. Instr. and Meth. A 356 (1995) 36

[38] B. van den Brandt et al., Nucl. Instr. and Meth. A 381 (1996) 219

[39] S. Reeve et al., Polarisation and relaxation characteristics of irradiated polmeric materials at $1 \mathrm{~K}$ and at $2.5 \mathrm{~T}$, in these proceedings

[40] L. Wang et al., DNP measurements of irradiated deuterated polyethylene, in these proceedings 